\title{
Preservation of Rear Wing with a Subvalvular Structure in Mitral Prosthetics
}

\author{
Zhumabaev Sultanmurat Abdiraimovich*, Asanaliev Mirlan Isaevich, \\ Urmanbetov Kubatbek Samyybekovich, Tursunbekova Gulnaz Tursunbekovna
}

Research Institute of Cardiovascular Surgery and Organ Transplantation of the Ministry of Health of the Kyrgyz Republic, Bishkek, Kyrgyzstan

\author{
Email address: \\ Sultanmurat-89@mail.ru (Z. S. Abdiraimovich), asanaliev80@gmail.com (A. M. Isaevich), urmankg@yandex.ru (U. K. Samyybekovich), \\ gulnaztursunbekova@gmail.com (T. G. Tursunbekovna) \\ *Corresponding author
}

\section{To cite this article:}

Zhumabaev Sultanmurat Abdiraimovich, Asanaliev Mirlan Isaevich, Urmanbetov Kubatbek Samyybekovich, Tursunbekova Gulnaz Tursunbekovna. Preservation of rear wing with a subvalvular structure in mitral prosthetics. Cardiology and Cardiovascular Research. Vol. 4, No. 4, 2020, pp. 175-179. doi: 10.11648/j.ccr.20200404.12

Received: September 12, 2020; Accepted: September 24, 2020; Published: October 7, 2020

\begin{abstract}
The goal is to evaluate the effectiveness of the immediate results (not more than 6 months) of the mitral valve prosthetics operation with preservation of the posterior valve with the chordal-papillary apparatus. Material and methods: the study included 96 patients after surgery with preservation of the posterior mitral valve cusp. The average age of the patients was $45.9 \pm 12.0$ years. The inclusion criteria were the final diastolic size (CRD) of the left ventricle (LV) and ejection fraction. Results: in the immediate postoperative period was observed a decrease in the final systolic and diastolic sizes and volumes of the left ventricle. A similar dynamics was noted in relation to the size of the left atrium. The pressure gradient on the mitral prosthesis and the systolic pressure in the pulmonary artery decreased. The ejection fraction of the left ventricle in the near postoperative period increased on average $57.9 \pm 9.8$ before surgery and $59.2 \pm 8.2$ after surgery. According to echocardiography, there were no cases of dysfunction of the artificial heart valve or prosthesis-dependent complications due to preserved cusp tissue or chords of the posterior cusp, and there was a positive dynamics of remodeling of the left ventricle. The surgical technique of preserving the posterior valve with its chordal-papillary apparatus is simple in surgical execution and does not lengthen the time of cardiopulmonary bypass $(57.3 \pm 26.1 \mathrm{~min})$.
\end{abstract}

Keywords: Mitral Valve Replacement, Subvalvular Structure, Posterior Leaflet, Left Ventricle

\section{Relevance}

Mitral valve replacement (MVR), both in the past and in the present, is the main radical treatment for acquired mitral valve defects. MVR leads to an improvement in intracardiac hemodynamics, a decrease in pulmonary arterial pressure, an improvement in the functional capabilities of patients, and a decrease in drug administration. At the same time, a number of unresolved problems remain: with an implanted prosthesis, the geometry of the left ventricle (LV) changes, myocardial contractility decreases [1-3].

As far back as the 1960-70s, it was shown that the preservation of the chord-papillary apparatus of Mitral valve (MV) and annulopapillary continuity plays a crucial role in the functioning of the LV. Many surgeons believe that the preserved chord-papillary apparatus of MV allows you to leave the sequence of physiological contraction of the left ventricular wall unchanged, improves contractility of the LV myocardium in the near and long-term postoperative period, especially in patients with increased LV and mitral insufficiency [4-6].

Preservation of subvalvular structures is not a new concept, however, which methods of surgical treatment of mitral defect, depending on the type of valve damage and subvalvular structures, should be adopted to achieve the best immediate clinical results, have not been conclusively defined and are debatable [9-12]. Therefore, the need for complete removal or preservation of valves, subvalvular structures of $\mathrm{MV}$, their role in changing the functional state of the myocardium is still under discussion. 
The aim of the study was to demonstrate the feasibility of preserving the posterior leaflet of MV with a chordicpapillary apparatus in patients with MVR.

\section{Material and Methods}

\subsection{Material}

From January 2004 to February 2018, 96 MVR operations with posterior leaflet preservation of mitral valve with chordalpapillary apparatus (CPA) were performed at the Science Research Institute of Heart Surgery and Organ Transplantation in patients with MV defects. The average age of the patients was $45.9 \pm 12$ years. $66(68.75 \%)$ were female patients and 30 $(31.25 \%)$ male patients. All patients had heart failure of various severity -2 functional class (FC) there were $8(8.3 \%)$, in $3 \mathrm{FC}-69(71.87 \%)$ and in $4 \mathrm{FC}-19(19.79 \%)$ patients. In 50 $(52.1 \%)$ cases before surgery, there was atrial fibrillation.

In the overwhelming majority, the cause of MV defect was rheumatism (88 (91.6\%) patients), 8 (8.3\%) had congenital MV insufficiency, and closed mitral commissurotomy was performed in 18 patients. Mitral stenosis or its predominance was diagnosed in 61 patients, insufficiency or its predominance was noted in 35 (table 1).

Clinical characteristics of patients table 1.

Table 1. Clinical characteristics of patients

\begin{tabular}{lll}
\hline Indicators & Absolute Number & $\%$ \\
\hline Average age, years & $45.9 \pm 12.0$ & \\
Sex Male & 30 & 31.25 \\
Female & 66 & 68.75 \\
Functional class: & & \\
II & 8 & 8,3 \\
II & 69 & 71,87 \\
IV & 12 & 19,79 \\
Reason for defect & & \\
Rheumatism & 88 & 91,6 \\
Congenital defect & 8 & 8,3 \\
Heart rhythm: & & \\
sinus rhythm & 46 & 47,9 \\
Atrial fibrillation & 50 & 52,1 \\
The nature of mitral valve disease: & & \\
stenosis & 40 & 41,6 \\
predominance of stenosis & 21 & 21,8 \\
failure & 19 & 19,79 \\
prevalence of failure & 16 & 16.6 \\
Cardiothoracic index & $60 \pm 9,1$ & - \\
Moore Index & $34.3 \pm 5$ & - \\
Valve calcification & 14 & 14.58 \\
\hline
\end{tabular}

\subsection{Methods}

Echocardiographic examination (EchoCG) was the main method in the diagnosis of mitral valve lesions. Based on the data of the echocardiography method, it was possible to judge the structure and morphological changes of intracardiac structures, the degree of hemodynamic disturbances, which is necessary when deciding on the selection of a surgical method for the treatment of mitral heart disease. With EchoCG, attention is paid to the length of the chords, hypertrophy of the heads of the papillary muscles, the degree of subvalvular stenosis. The main indicators of hemodynamics and myocardial contractility were evaluated. Echocardiography data made it possible to suggest the type of surgery to be performed and to conduct postoperative assessment of the results of the performed intervention. In the postoperative period, the work of artificial prostheses, the presence of pathological flows, the dynamics of the size of the cavities of the heart and intracardiac hemodynamics were evaluated.

\subsection{Operational Technique}

Access to the heart was accomplished by anterior longitudinal sternotomy. Cardiopulmonary bypass was in accordance with the generally accepted pattern in conditions of normal or moderate hypothermia. Myocardial protection was carried out by cold hyperpotassium blood cardioplegia. After a visual assessment of the size of the atria, access to the mitral valve was selected. In the presence of malformation of the tricuspid valve, access was through the right atrium and atrial septum, and with isolated malformation of the MV through the left atrium. After examining the MV, a decision was made to replace the mitral valve with full or partial preservation of the valves and subvalvular apparatus (Figure 1).

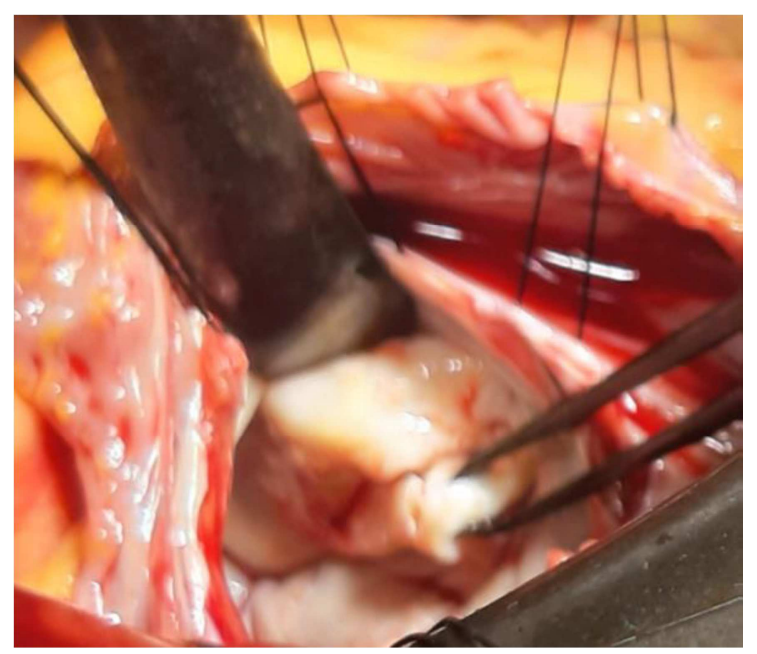

Figure. 1. Mitral valve before surgery.

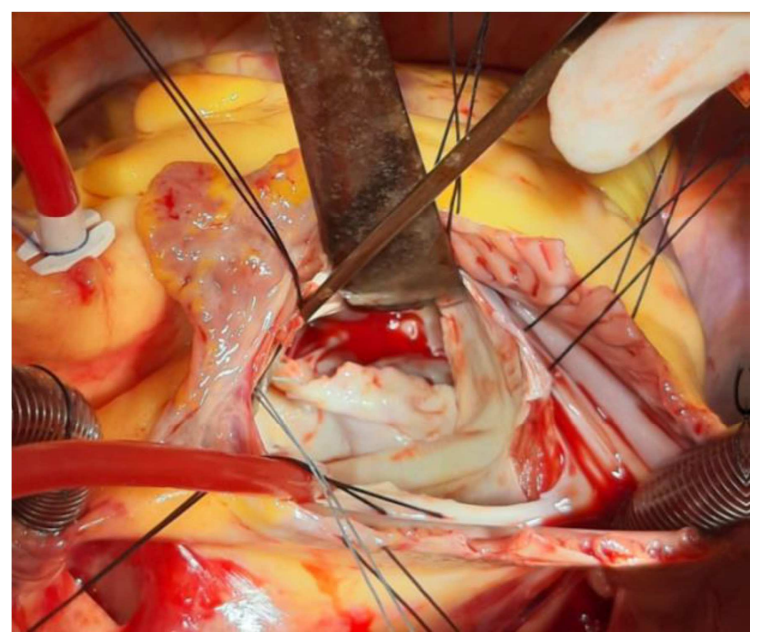

Figure 2. Preservation of the posterior cusp of mitral valve (plication method). 
If there was no gross calcification or gross fibrosis of the cusp, a sharp thickening and shortening of the papillary muscles or chord, then a decision was made to replace the valve while preserving the posterior cusp and subvalvular structures. In the presence of fusion of the valves in the area of the commissures, the commissures were dissected to the native fibrous ring. After that, the anterior leaflet with its chords from the inner commissure to the outer commissure was completely excised, leaving $2 \mathrm{~mm}$ of tissue of the anterior leaflet (Figure 2). At the same time, they captured part of the posterior leaflet before attaching the main chords.

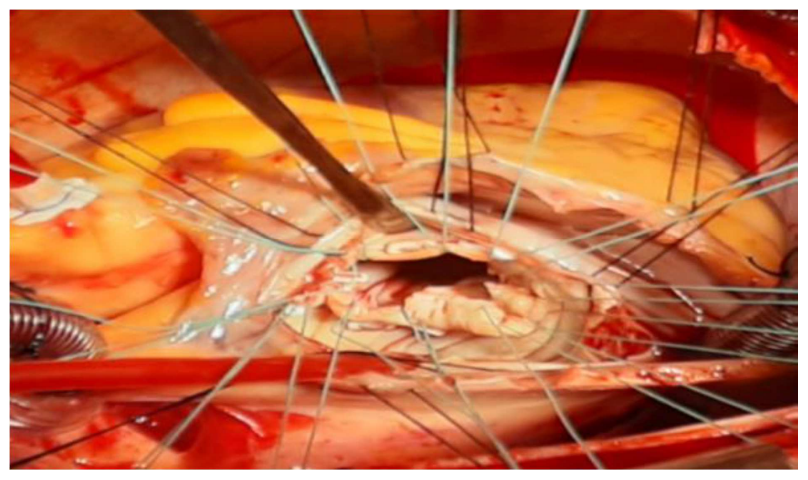

Figure 3. The imposition of I''shaped seams.

This tactic allows you to choose a large prosthesis and is easy to implant on the fibrous ring. A thorough examination of the posterior cusp, chords, and papillary muscles was then performed. The prosthetic calibrator measured the size of the fibrous ring, which should freely pass through the hole MV. The valve prosthesis was selected. In most cases, Sent-Jude No. 29 or OnX No. 29/31 double-wing prostheses were able to be implanted; in only three cases, No. 27 prosthesis. To fix prosthesis to the fibrous ring, a continuous suture technique was used with one or two threads and / or separate " $\Pi$ " shaped sutures (Figure 3). The orientation of bivalve prostheses was carried out in such a way that the half-disks with their apex coincided with the commissures of MV. The discs are checked for free movement after the prosthesis is completely fixed to the fibrous ring, (Figure 4).

Concomitant pathology was corrected simultaneously with MVR: annuloplasty of the tricuspid valve (according to Boyd, De-Vega) in 50 patients $(53.2 \%)$, thrombectomy from the left atrial cavity - in $11(11.5 \%)$, and atrioplasty of the left atrium according to Kawazoe - in 7 patients (7.4\%).

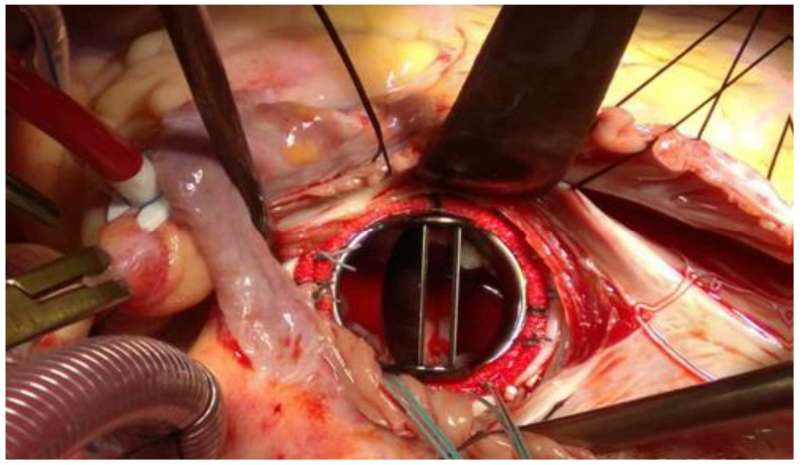

Figure 4. After complete fixation of the mitral prosthesis.

\section{Results and Discussion}

After removal of the MV anterior cusp, good access opens to assess the condition of the posterior cusp and subvalvular structure, and also makes it possible to select the type of suture technique for implanting an artificial valve (AV) into the fibrous ring.

When analyzing the study group by the type of mitral defect in patients with mitral insufficiency, the technique of complete conservation of the leaflet and chordal-papillary apparatus was more often used. Thickened chords along with papillary muscles can interfere with blood flow to the left atrium and create a large gradient between the left atrium and ventricle during diastole, therefore, in patients with severe mitral stenosis, in some cases they were forced to remove chords of the first order (Table 2).

Table 2. Types of mitral prosthesis operations with preservation of subvalvular structures of posterior leaflet

\begin{tabular}{llll}
\hline № & Preservation of subvalvular structures & Absolute number \\
\hline 1 & Complete preservation of the chordal-papillary apparatus & \% & 89 \\
2 & Partial preservation of the chordal-papillary apparatus & 94.7 & 5 \\
\hline
\end{tabular}

Complete preservation of the subvalvular structure prevailed of all the types of operations with the preservation of the posterior leaflet in $89(94.7 \%)$ cases, Due to the sharp thickening and shortening of the chords in $5(5.3 \%)$ cases, we had to remove the first-order chords. The amount of chord removal depended on the degree of thickening and shortening of the chordal-papillary apparatus. Of these, in 1 case two were deleted and in 4 - one first-order chord. Nevertheless, being of the opinion that the preserved chordal-papillary apparatus of MK leads to an improvement in myocardial function, it is necessary to completely save the leaflet with subvalvular apparatus [13-14].
Table 3. Surgical indications.

\begin{tabular}{ll}
\hline The average time of cardiopulmonary bypass, min & $84,7 \pm 29,8$ \\
Average aortic clamping time, min & $57,3 \pm 26,1$ \\
Cardiac restoration: & \\
Self & 54 \\
Through fibrillation & 20 \\
Via AB block & 22 \\
\hline
\end{tabular}

The duration of cardiopulmonary bypass was on average $84.7 \pm 29.8$ minutes, the aortic clamping time was $57.3 \pm 26.1$ minutes (Table 3 ). Cold hyper potassium cardioplegia with blood was used to protect myocardium.

$32(33.3 \%)$ patients had various types of complications in 
the postoperative period of 96 patients, (table 4).

Table 4. Postoperative complications.

\begin{tabular}{llll}
\hline № & Types of Complications & Absol. number & \% \\
\hline \multirow{4}{*}{1} & Fatal complications: & 4 & \\
& a) heart failure & 2 & 4,2 \\
& b) respiratory failure & 1 & \\
& c) multiple organ failure & 1 & \\
& Nonfatal complications: & 28 & 29,2 \\
2 & a) heart failure & 26 & \\
& b) bleeding & 1 & \\
& c) mediastinitis & 1 & \\
\hline
\end{tabular}

Postoperative mortality was $4.2 \%$. Fatal outcomes were not associated with the applied MVR technique.

Nonfatal postoperative complications were noted in 28 cases. Cardiotonics were used in therapeutic doses in patients with heart failure, only in three cases were they used higher therapeutic doses and prolonged mechanical ventilation.

A patient with postoperative bleeding underwent a resternotomy with a surgical stop. The patient was safely discharged home.

One patient developed serous-purulent mediastinitis after a previously performed closed mitral commissurotomy. The sternum osteosynthesis was performed, discharged on the 12th day after osteosynthesis.

Echocardiography is the main method for adequately assessing the effectiveness of the posterior leaflet preservation technique with the chordal-papillary apparatus. An analysis of the echocardiographic parameters of the studied patients showed the presence of the best positive dynamics in the immediate postoperative period (Table 5).

Table 5. Dynamics of echocardiography indicators in patients after mitral valve replacement.

\begin{tabular}{llll}
\hline Indicators & Before surgery & After operation & P \\
\hline LVd, cm & $5,5 \pm 0,9$ & $5,2 \pm 0,6$ & $<0,05$ \\
LVs, cM & $3,7 \pm 0,7$ & $3,5 \pm 0,7$ & $<0,05$ \\
EF, \% & $57,9 \pm 9,8$ & $59,2 \pm 8,2$ & $=0,05$ \\
LV EDV, ml & $143,7 \pm 57,2$ & $132,8 \pm 39,6$ & $<0,05$ \\
LV ESV, ml & $62,1 \pm 32,4$ & $55,9 \pm 27,7$ & $<0,05$ \\
SV, ml & $70,8 \pm 9,8$ & $71,9 \pm 19,8$ & $=0,05$ \\
LAs, cm & $5,4 \pm 1,4$ & $4,5 \pm 0,8$ & $<0,05$ \\
RVs, cm & $2,5 \pm 0,6$ & $2,4 \pm 0,3$ & $=0,05$ \\
Systolic PAP mm Hg & $59,7 \pm 16,7$ & $41,1 \pm 8,8$ & $<0,05$ \\
LV sphericity index & $0,72 \pm 0,1$ & $0,67 \pm 0,1$ & $<0,05$ \\
\hline
\end{tabular}

The table shows that in the postoperative period, the indicators changed slightly. Apparently, this is due to the fact that we did not begin to analyze separately patients with mitral stenosis or insufficiency. Nevertheless, there was a decrease in the size of the left parts of the heart, an increase in the contractility of the LV myocardium, a decrease in pulmonary hypertension and a decrease in the sphericity of the LV in patients after MVR with preservation of the posterior leaflet and chordal-papillary apparatus [15].

According to echocardiography, there were no cases of dysfunction of the artificial heart valve or prostheticdependent complications due to the left leaf tissue or the subvalvular structure of the MV posterior leaflet.

\section{Conclusion}

Thus, according to the data obtained as a result of the analysis of the immediate postoperative results, good results were found for prosthetic surgery with preservation of the MV posterior leaflet and subvalvular structures compared to standard prosthetics, since the radical removal of the valve apparatus is a factor in a significant decrease in LV function.

The surgical technique for maintaining the posterior leaflet with its subvalvular structure is simple in surgical execution and does not lengthen the time of cardiopulmonary bypass.

\section{References}

[1] Shumakov V. I., Semenovskiy M. L., Sokolov V. V. Mitral valve replacement with full or partial preservation of subvalvular structures. // Thoracic surgery. 1989. №.3. P. 5-9.

[2] Yilong Guo, Shuwu He, Tianguang Wang, Comparison of modified total leaflet preservation, posterior leaflet preservation, and no leaflet preservation techniques in mitral valve replacement - a retrospective study. J Cardiothorac Surg 2019; $14: 102$.

[3] Goldman M. E., Mora F., Guarino T., Fuster V., Mindich BP. Mitral valvuloplasti is superior to valve replacement for preservation of left ventricular function. An intraoperative two-demontional echocardiographic study// J. Amer. Coll. 1987. V. 10. N 3. P. $568-575$.

[4] Lillechei C. W., Levy M. J. Mitral valve replacement with preservation of papillary muscles and chordae tendinea // J. Thorac. Cardiovasc. Surg. 1964. Vol. 47, N 4. P. 532-543.

[5] Toktosunova D. B., Djundubaev M. K., Seytahunova E. D., Ahmedova I. A. Remodeling of the left heart in patients with rheumatic mitral valve disease after mitral valve replacement with preservation of subvalvular structures // J. Scientific Research Institute of Heart Surg. and Organ Transplantation. 2020. 4. 40-44.

[6] Shevchenko Y. L., Popov L. V. Volkova L. V. 20-years' experience of MVR with full or partial preservation of valve structures. // Bulletin of NCCVS named. A. N. Bakulev RAMS. 2006. T. 7, №3. P. 41.

[7] Sintek C. F., Khonsari S. Use of extended polythetrafluoroethylene (ePTFE) chordae to re-establish annular-papillary connection after mitral valve excision // J. Heart Valve Dis. 1996. Vol. 5. P. 362-364.

[8] Ivanov V. A. Popov S. O., Kashin V. Y., Konstantinov B. A. Subvalvular structures preservation at mitral valve replacement. //Surgery Journal named. N. I. Pirogov. 2007. №7. P. 36-40.

[9] Chen L., Chen B., Hao J., Wang X., Ma R., Cheng W., Qin C., Xiao Y. Complete preservation of the mitral valve apparatus during mitral valve replacement for rheumatic mitral regurgitation in patients with an enlarged left ventricular chamber //Heart Surg. Forum. 2013. V. 16. N3. P. 137-43.

[10] Dobrotin S. S. Surgical treatment of mitral stenosis and its complicated forms. / Dobrotin S. S., Gamzaev A. B., Chiginev V. A. Lashmanov D. I. //Thoracic and Cardiovascular surgery. 2005. - № 3. - P. 15-19. 
[11] Athanasiou T., Chow A., Rao C., Aziz O., Siannis F., Ali A., Darzi A., Wells F. Preservation of the mitral apparatus: evidence synthesis and critical reappraisal of surgical techniques //Eur. J. Cardiotorac Surg. 2008. V. 33. N3. P. 391401.

[12] Casquero E., Asorey V., Lugo J., Pradas G. Ruptured papillary muscle after mitral valve replacement with preservation of subvalvular structures //J. Cardiol. Surg. 2010. V. 25. N 6. P. 694-695.

[13] Coutinho GF, Bihun V, Correia PE, Antunes PE, Antunes MJ. Preservation of the subvalvular apparatus during mitral valve replacement of rheumatic valves does not affect long-term survival. //Eur J, Cardiothorac, Surg. 2015. V. 48. N6. P. 8617.

[14] Lafci G., Cagli K., Cicek O. F., Korkmaz K., Turak O., Uzun A., Yalcinkaya A., Diken A., Gunertem E. Papilary muscle repositioning as a subvalvular apparatus preservation technique in mitral stenosis patients with normal left ventricular systolic function //Tex Heart Inst J. 2014. V. 41. N1. P. 33-39.

[15] Timala RB, Joshi D, Aryal M, Bhandari K, Singh Y, Sharma J. Effects of subvalvular apparatus preservation in mitral valve replacement among rheumatic patients: early and mid-term follow up. J Inst Med 2016, 38: 2-3.

\section{Biography}

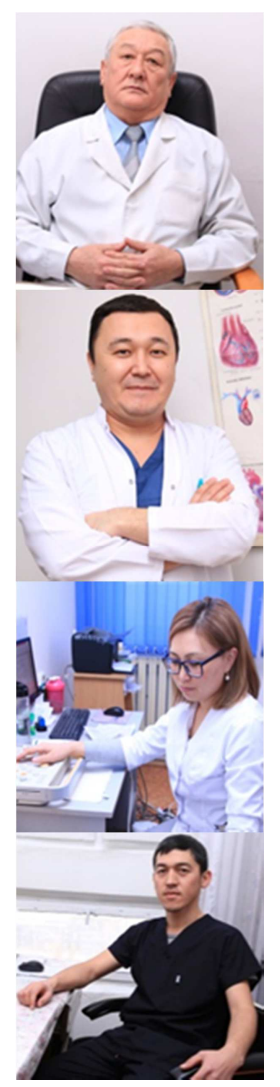

Urmanbetov Kubatbek Samyybekovich doctor of medical Sciences, leading researcher At the research Institute of heart surgery and organ transplantation.

Asanaliev Mirlan Isaevich, $\mathrm{PhD}$, research associate At the research Institute of heart surgery and organ transplantation. Cardiosurgeon of the Department of coronary surgery and surgery of acquired heart defects.

Tursunbekova Gulnaz Tursunbekovna doctor of functional diagnostics of the consultative and diagnostic Department of the Research Institute of heart surgery and organ transplantation.

Zhumabaev Sultanmurat Abdiraimovich is a Junior researcher at the research Institute of heart surgery and organ transplantation. 